\title{
Measurement of total bile acids in gastric juice
}

\author{
BJ COLLINS, ${ }^{*}$ PCH WATT, $\dagger$ THERESA O' REILLY, $\dagger$ RJ McFARLAND $\ddagger$ AHG LOVE*
}

From the *Department of Medicine, the †Department of Surgery, the Queen's University of Belfust and $\ddagger$ Ulster Hospital, Dundonald, Belfast

SUMMARY An established method for the assay of total bile acids was validated for use in fasting and post-prandial gastric juice samples. Fasting and post-prandial intragastric bile acid concentrations were measured in 29 healthy volunteers, 15 patients after vagotomy and gastrojejunostomy (V and GJ) and 15 patients after vagotomy and pyloroplasty ( $\mathrm{V}$ and $\mathrm{P}$ ). Healthy female volunteers had higher post-prandial bile acid concentrations than age matched healthy males $(p<0 \cdot 02)$. Patients with V and GJ had higher fasting and post-prandial bile acid concentrations than age and sex matched control subjects $(p<0 \cdot 01)$. Patients with $V$ and $P$ had higher bile acid concentrations than control subjects only in post-prandial samples $(p<0.05)$.

Gastric juice contamination by bile acid has been implicated in the pathogenesis of gastritis, ${ }^{1}$ gastric ulcer $^{2}$ and reflux oesophagitis. ${ }^{3}$ Duodenogastric bile reflux may also promote carcinogenesis in the stomach. ${ }^{4}$

Fausa and Skalhegg have described a simple method for the assay of total bile acid in duodenal aspirates. ${ }^{5}$ This method has the advantage of not requiring extraction or protein precipitation prior to analysis. However, the technique has not been validated for samples of gastric juice. Separate validation for the analysis in gastric juice is necessary since there are marked differences between duodenal and gastric juice, particularly with regard to $\mathrm{pH}$. In addition, a corn oil test meal is often used to stimulate bile flow in the assessment of post-prandial duodenogastric reflux. There is currently no information on the effect of corn oil contamination of gastric juice on the assay. We assessed the use of Fausa and Skalhegg's method for the measurement of bile acid concentration in fasting and postprandial gastric juice samples in normal subjects and in a series of post-gastric surgery patients.

\section{Subjects and methods}

Fifty nine subjects ( 24 men and 35 women) whose ages ranged from 21 to $67 \mathrm{yr}$ (mean $51.4 \mathrm{yr}$ ) were studied. Twenty nine volunteers had no previous gastric surgery and had no past or present symptoms of gastrointestinal tract disease. Fifteen patients previously had a vagotomy and gastrojejunostomy (V and GJ) and 15 had vagotomy and pyloroplasty ( $V$ and $P$ ). All the surgery was carried out between 1957 and 1967 for duodenal ulcer. None of the post-gastric surgery patients had symptoms suggestive of duodenogastric bile reflux.

Informed consent was obtained from all subjects and ethical committee approval was granted for the study.

Patients and volunteers had a size 14 Salem sump, nasogastric tube passed after an overnight fast. The position of the nasogastric tube was checked radiologically. After 35-45 min the stomach was completely aspirated and the juice discarded. Gastric juice was then collected over a $30 \mathrm{~min}$ period. A meal containing $18 \mathrm{~g}$ corn oil and $12 \mathrm{~g}$ glucose made up to $100 \mathrm{ml}$ with warm water was given via the nasogastric tube over a period of two minutes. Aliquots of gastric juice were then aspirated at 20, 40 and $60 \mathrm{~min}$ after the meal. All gastric juice samples were centrifuged at $1500 \mathrm{~g}$ and $4^{\circ} \mathrm{C}$ for $15 \mathrm{~min}$ to separate mucus and corn oil from the aqueous portion.

The analysis of total bile acid was carried out using reagent mixtures as described for the commercial kit produced by Nyegaard (Sterognost $3 x$ Pho) which is based on the Fausa and Skalhegg method. ${ }^{5}$ This technique involves the oxidation of bile acids by the enzyme $3 x$ hydroxysteroid dehydrogenase with concomitant reduction of NAD to $\mathrm{NADH}_{2}$. The $\mathrm{NADH}_{2}$ is produced mole for mole from bile acid and can be readily quantified using a spectrophotometer (Pye Unicam SP8-100) set to measure absorbance (A) at $340 \mathrm{~nm}$. Extraction of bile acids from gastric juice into methanol and 
Table 1 Control solutions for bile acid assay

\begin{tabular}{llllll}
\hline Tube & Reagent mixture $(\mathrm{ml})$ & Hydrazine hydrate $(\mathrm{ml})$ & Methanol $(\mathrm{ml})$ & Sample $(\mu \mathrm{l})$ & Saline $(\mu \mathrm{l})$ \\
\hline 1 & 1.25 & - & 0.25 & 100 & - \\
2 & -1.25 & - & 0.25 & 100 & - \\
3 & 1.25 & 1.25 & 0.25 & - & 100 \\
4 & - & & 0.25 & 100 & \\
\hline
\end{tabular}

Corrected $A=\left(A_{1}-A_{2}\right)-\left(A_{3}-A_{4}\right)$

protein precipitation are not required in this method of measuring bile acid concentration.

In the assay, $100 \mu \mathrm{l}$ gastric juice and $0.25 \mathrm{ml}$ methanol were added to $1.25 \mathrm{ml}$ of a reagent mixture containing NAD $(0.4 \mathrm{mmol} / \mathrm{l})$, sodium pyrophosphate $(52 \mathrm{mmol} / \mathrm{l})$, enzyme $(0.07 \mathrm{IU} / \mathrm{ml})$, hydrazine hydrate $(0.1 \mathrm{~mol} / \mathrm{l})$ and EDTA, sucrose and dithiothreitol as stabilising agents. The solution was mixed thoroughly and incubated at room temperature for $20 \mathrm{~min}$ before reading the absorbance in the spectrophotometer. A series of control solutions were assayed in each run to correct for absorbance due to other constituents of the reaction mixture (Table 1). Each gastric juice sample was assayed in triplicate and three standard solutions of cholic acid were analysed with each run of samples to check the accuracy of the assay. Bile acid concentrations were calculated from the absorbance reading using the millimolar extinction coefficient of $\mathrm{NADH}_{2}$ at $340 \mathrm{~nm}(6 \cdot 22 \mathrm{mmol} / \mathrm{l} / \mathrm{cm})$.

Thus, bile acid concentration $(\mu \mathrm{mol} / \mathrm{l})=$

$$
\frac{\mathrm{A} \times 1.6 \times 1000}{6.22 \times 0.1}
$$

where $1.6 \mathrm{ml}=$ volume of total reaction mixture and $0 \cdot 1 \mathrm{ml}=$ volume of gastric juice sample.

All statistical analyses were carried out using the Wilcoxon signed rank test.

\section{Results}

In our laboratory we found that the reaction was complete after $15 \mathrm{~min}$ when the assay was applied to a bile stained sample of gastric juice. The assay was linear (coefficient of correlation 0.999) for cholic acid standard aqueous solutions, up to a concentration of $1000 \mu \mathrm{mol} / \mathrm{l}$, when using $100 \mu \mathrm{l}$ sample volumes. A series of dilutions was prepared from a bile stained sample of gastric juice (concentration = $1000 \mu \mathrm{mol} / \mathrm{l}$ ) and a linear relation was found between the absorbance readings and the bile acid concentration over the range of dilutions tested (correlation coefficient 0.992 ). Thereafter, heavily bile stained gastric juice samples were diluted with normal saline until the concentration was less than $1000 \mu \mathrm{mol} / \mathrm{l}$.

To assess reproducibility, 12 aliquots of a mini- mally bile stained sample of gastric juice ( $\mathrm{pH} \mathrm{2 \cdot 4)}$ were assayed. The concentration was found to be $108.03 \mu \mathrm{mol} / \mathrm{l}$ and the coefficient of variation was $3.7 \%$. The same gastric juice was then spiked with cholic acid in order to raise the bile acid concentration by $200 \mu \mathrm{mol} / \mathrm{l}$. Twelve aliquots were assayed giving a mean value of $284.8 \mu \mathrm{mol} / \mathrm{l}$ (coefficient of variation $2.02 \%$ ). The recovery of cholic acid was $90.4 \%$.

The effect of gastric juice $\mathrm{pH}$ on the assay was assessed. A sample of bile stained gastric juice of $\mathrm{pH}$ 6.34 and bile acid concentration of $530.0 \mu \mathrm{mol} / \mathrm{l}$ was divided into 7 aliquots. The aliquots were acidified with $6 \mathrm{M}$ hydrochloric acid to produce a range of $\mathrm{pH}$ values from 0.97 to 6.34 . Each aliquot was assayed in triplicate and results (after correction for dilution with hydrochloric acid) expressed as a percentage of the bile acid concentration in the original sample (Table 2).

The effect of corn oil contamination of postprandial samples was assessed. A sample of bile stained gastric juice was divided into two $5 \mathrm{ml}$ aliquots ( $\mathrm{A}$ and $\mathrm{B}$ ). To aliquot $\mathrm{A}, 5 \mathrm{ml}$ of corn oil were added and the gastric juice and corn oil were thoroughly mixed with a vortex mixer. Aliquot B was unaltered. Both aliquots were allowed to rest in a water bath at $37^{\circ} \mathrm{C}$ for one hour. Both samples were then centrifuged at $1500 \mathrm{~g}$ at $4^{\circ} \mathrm{C}$ for $15 \mathrm{~min}$. After centrifugation the aqueous phase of aliquot $\mathrm{A}$ was separated from the corn oil and bile acid concentrations were determined for 12 samples of each aliquot. For aliquot $\mathbf{A}$ the mean concentration was $368.5 \mu \mathrm{mol} / \mathrm{l}$ (coefficient of variation $3.6 \%$ ) and for aliquot $B$ the mean concentration was $423.6 \mu \mathrm{mol} / \mathrm{l}$

Table 2 Bile acid concentration in gastric juice samples of different $\mathrm{pH}$ (expressed as a percentage of the concentration of the unaltered sample)

\begin{tabular}{ll}
\hline pH of gastric juice aliquot & $\begin{array}{l}\text { Bile acid content } \\
\text { (\% of concentration of unaltered } \\
\text { sample) }\end{array}$ \\
\hline 6.34 & 100.0 \\
4.35 & 98.7 \\
2.95 & 104.8 \\
2.30 & 101.7 \\
1.82 & 98.6 \\
1.28 & 97.0 \\
0.97 & 100.2 \\
\hline
\end{tabular}


Table 3 Comparison of intragastric bile acid concentrations ( $\mu \mathrm{mol} / \mathrm{l})$ in age matched men and women control subjects

\begin{tabular}{|c|c|c|c|}
\hline \multirow[b]{3}{*}{$\begin{array}{l}\text { Fasting } \\
\text { Peak post-prandial }\end{array}$} & \multicolumn{3}{|c|}{ Median (range) concentrations ( $\mu \mathrm{mol} / \mathrm{l})$} \\
\hline & $\operatorname{Men}(n=1$ & & Women $(n=12)$ \\
\hline & $\begin{array}{l}0(0-484) \\
10(0-72)\end{array}$ & $\begin{array}{l}\text { NS } \\
\mathrm{p}<0.02\end{array}$ & $\begin{array}{c}1(0-301) \\
34(0-1055)\end{array}$ \\
\hline
\end{tabular}

(coefficient of variation $2.7 \%$ ). Therefore, the contamination and subsequent removal of corn oil from gastric juice reduced the bile recovery by $13 \%$.

Of the 29 control subjects, 12 were men, (mean age $42.3 \mathrm{yr}$ ), these were age-matched to within $10 \mathrm{yr}$ with 12 of the women subjects (mean age $45.4 \mathrm{yr}$ ) and pre- and post-prandial gastric juice bile acid concentrations measured. Athough there were no significant differences between the groups for fasting samples, women had significantly higher peak postprandial bile acid concentrations (Table 3 ). The $\mathrm{V}$ and $\mathrm{GJ}$ (mean age 55.6 yr) and $\mathrm{V}$ and $\mathrm{P}$ (mean age $56 \cdot 1 \mathrm{yr}$ ) patients were sex and age matched to within

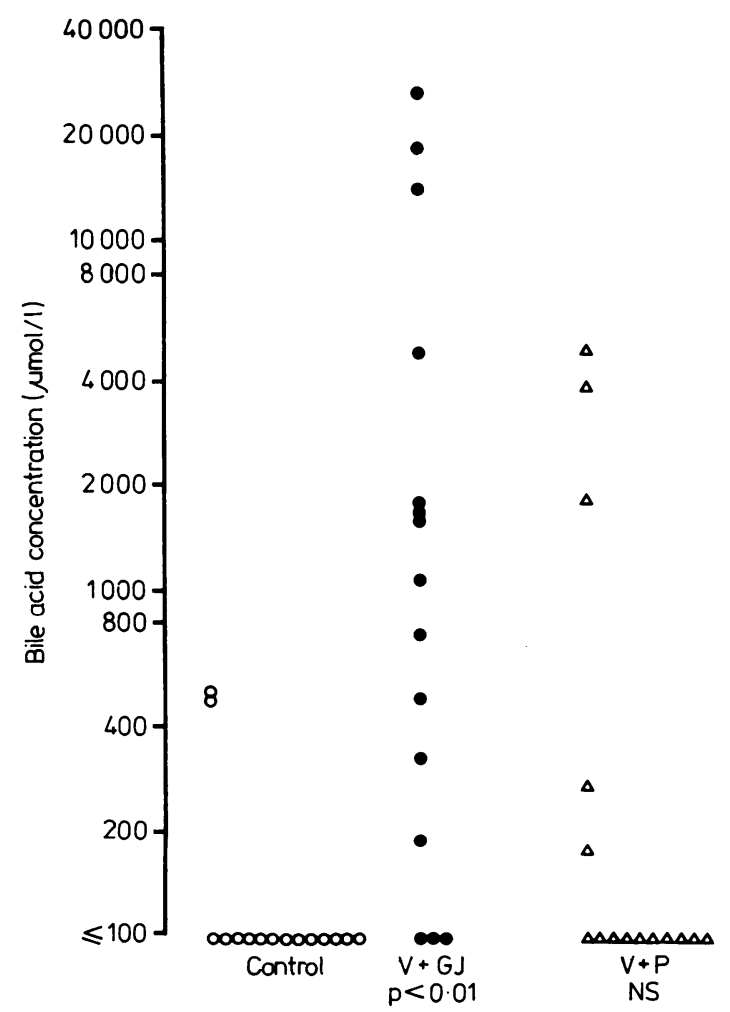

Fig. 1 Fasting intragastric bile acid concentrations in control subjects, vagotomy and gastrojejunostomy $(V+G J)$ and vagotomy and pyloroplasty $(V+P)$ patients.

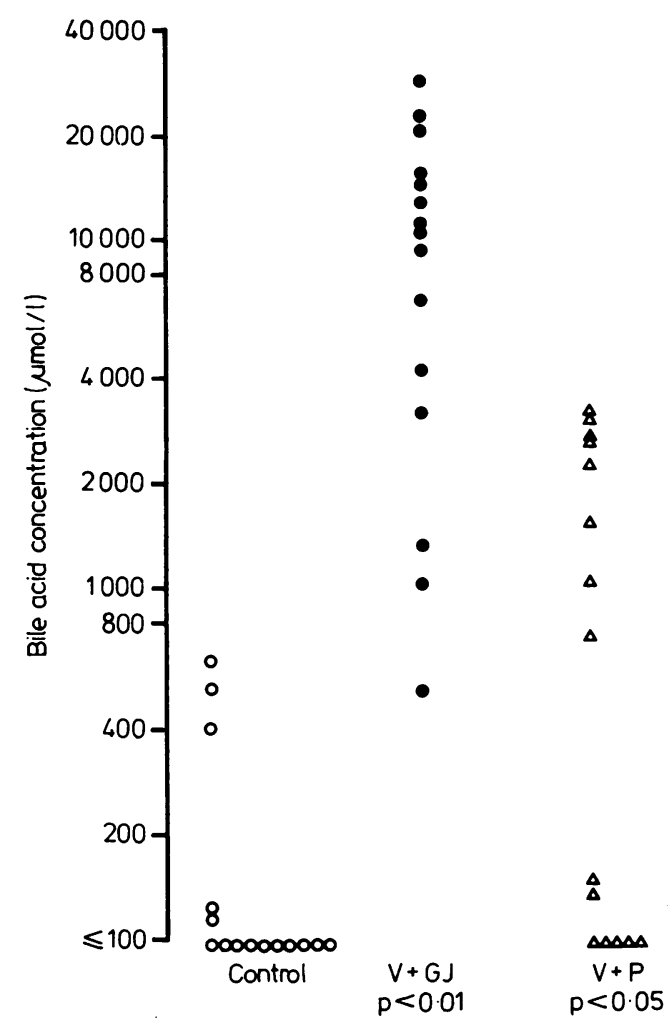

Fig. 2 Peak post-prandial intragastric bile acid concentrations in control subjects, vagotomy and gastrojejunostomy $(V+G J)$ and vagotomy and pyloroplasty $(V+P)$ patients.

10 years of each other. Both groups were also sex and age matched to within $10 \mathrm{yr}$ with 15 of the control subjects (mean age of this group 53.3 yr). Patients who had previous V and GJ had significantly higher fasting and peak post-prandial gastric bile acid concentrations than controls. However, the $V$ and $P$ patients only had significantly increased bile acid concentrations post-prandially (Figs. 1 and 2). Maximum post-prandial intragastric bile acid concentrations were detected at $20 \mathrm{~min}$ in two control subjects, four $\mathrm{V}$ and GJ patients and three $\mathrm{V}$ and $\mathrm{P}$ patients; at $40 \mathrm{~min}$ in one control, seven $V$ and $G J$ patients and two $V$ and $P$ patients; at $60 \mathrm{~min}$ in nine controls, four $\mathrm{V}$ and GJ patients and six $V$ and $P$ patients. Three control subjects and four $V$ and $P$ patients had no detectable bile acid in post-prandial samples.

\section{Discussion}

Our data show that the method used by Fausa and 
Skalhegg is suitable for measurement of total bile acid concentration in gastric juice. In particular, $\mathrm{pH}$ does not appear to influence results although we noted that contamination of gastric juice with corn oil reduced recovery of bile acids.

We have demonstrated a difference in postprandial gastric bile acid concentrations between men and women control subjects. To our knowledge this has not been previously reported and it highlights the need for careful sex matching in studies of duodenogastric reflux. A surprisingly wide interindividual range of gastric bile acid concentration was found among control subjects. Even more striking variation was found in the operated subjects. This observation suggests that considerable duodenogastric reflux of bile can occur in normal subjects and in post-gastric surgery patients without the production of symptoms.

The differences between men and women and between control subjects and $V$ and $P$ patients were found only in the post-prandial samples. This emphasises the necessity to stimulate bile flow with a test meal in the study of duodenogastric reflux.

BJ Collins and PHC Watt were in receipt of Royal Victoria Hospital Research Fellowships during the period of this work.
We thank Professor DT Burns, Professor of Analytical Chemistry, The Queen's University of Belfast, who advised on the final manuscript.

\section{References}

' Ritchie WP. Alkaline reflux gastritis. An objective assessment of its diagnosis and treatment. Ann Surg 1980;192:288-98.

${ }^{2}$ Black RB, Roberts G, Rhodes J. The effect of healing on bile reflux in gastric ulcer. Gut 1971;12:552-8.

${ }^{3}$ Crumplin MKH, Stol DW, Murphy GM, Collis JL. The pattern of bile salt reflux and acid secretion in sliding hiatal hernia. $\mathrm{Br}$ J Surg 1974;61:611-6.

${ }^{4}$ Domellof L. Gastric carcinoma promoted by alkaline reflux gastritis-with special reference to bile and other surfactants as promoters of post-operative gastric cancer. Medical Hypotheses 1979;5:463-76.

${ }^{5}$ Fausa O, Skalhegg BA. Quantitative determination of bile acids and their conjugates using thin layer chromatography and a purified $3 x$-hydroxysteroid dehydrogenase. Scand J Gastroenterol 1974;9:249-54.

Requests for reprints to: Dr BJ Collins, Department of Medicine, The Queen's University of Belfast, Grosvenor Road, Belfast BT12 6BJ, N Ireland. 